Reprod. Nutr. Dévelop., 1981, 21 (4), 497-504.

\title{
Digestibilité apparente après ligature du canal pancréatique chez le lapin en croissance : effet d'une alimentation ad libitum
}

\author{
par C. SIMOES NUNES, F. LEBAS *,T. CORRING \\ avec la collaboration technique de Dominique PILLOIS ef Y. LEMAIRE. \\ Laboratoire de Physiologie de la Nutrition, I.N.R.A. \\ 78350 jouy en Josas, France. \\ * Laboratoire de Recherches sur l'Elevage du Lapin, I.N.R.A. \\ B.P. 12, 31320 Castanef Tolosan, France.
}

Summary. Pancreatic duct ligature in the growing rabbit fed ad libitum.

We studied the apparent digestibility of an ad libitum diet in 16 growing rabbits, 8 with pancreatic duct ligature $\left(\mathrm{P}^{-}\right)$and 8 sham-operated (TO). The study was divided into two 10-day periods, starting 5 and 33 days, respectively, after surgery. The food intake of the sham-operated animals was higher $(160 \mathrm{~g} /$ day at the end of the experiment) than that of the ligated ones (stabilized at 100-120 g/day) ; mean daily gain was also higher in the former (TO : $33.7 \mathrm{~g} /$ day, $\mathrm{P}^{-}: 15.6 \mathrm{~g} /$ day ; $\mathrm{P}<0.01$ ). Dry matter $(\mathrm{MS})$ and energy digestibilities were not significantly different in the $\mathrm{P}^{-}$and the TO groups, but nitrogen $(\mathrm{N})$ digestibility, decreased after pancreatic duct ligature (TO : 86.1, $\mathrm{P}^{-}: 64.9 ; \mathrm{P}<0.01$ ), then increased in time much more in $P$ - than in sham-operated rabbits, but remained lower in the ligated group ( -5.6 points). The results suggest that digestive compensation was established after pancreatic duct ligature ; its origin is hypothesized.

\section{Introduction.}

La suppression de l'hydrolyse enzymatique pancréatique dans la lumière intestinale entraîne une diminution de la digestibilité apparente de l'aliment chez toutes les espèces étudiées (Sateri, 1975 ; Douglas ef al., 1953 ; Shingleton et al., 1955 ; Corring et Bourdon, 1976, 1977 ; Catala, 1976). Chez le lapin soumis à une alimentation restreinte (Corring et Lebas, 1977), la ligature du canal pancréatique diminue l'utilisation digestive apparente de l'azote de 27,6 p. 100 et celle de l'énergie de 3,9 p. 100. Parallèlement, les animaux privés de leur sécrétion pancréatique ont, comparés aux témoins, une croissance pondérale significativement plus faible. Il est logique de rapporter cette réduction de la vitesse de croissance à celle du flux quotidien de nutriments métabolisables, réduction entraînée par la diminution de l'efficacité de la digestion. Or, dans l'essai de Corring ef Lebas (1977), les animaux ne recevaient chaque jour que $100 \mathrm{~g}$ d'aliment ef n'avaient donc pas la possibilité de compenser la faible efficacité digestive par une surconsommation alimentaire. II a été en effet nettement 
démontré (Lebas, 1975 ; Bombeke, Okerman et Normans, 1978 ; Spreadbury ef Davidson, 1978 ; Dehalle, 1980) que les lapins nourris à volonté ajustent leur consommation d'aliment de manière à obtenir chaque jour la quantité d'énergie digestible nécessaire et assurent ainsi une croissance constante quelle que soit l'efficacité de la transformation digestive de l'énergie.

Il nous est donc apparu intéressant de chercher à savoir si, chez des lapins alimentés à volonté, après ligature du canal pancréatique, la digestibilité est autant affectée que chez les animaux restreints et si, le cas échéant, ces animaux savaient compenser la réduction de la transformation digestive par une surconsommation alimentaire leur permettant de maintenir leur croissance.

\section{Matériel et méthodes.}

Animaux ef alimentation. - Seize lapins des 2 sexes, de race Californienne ont été utilisés. Ils ont été placés à l'âge de 37 jours et au poids vif moyen de $0,98 \pm 0,02 \mathrm{~kg}$ dans des cages métalliques permettant la collecte totale des fèces et le contrôle de l'ingestion alimentaire. Tous les animaux ont été alimentés à volonté jusqu'à la fin de l'expérimentation, à l'exception du jour précédant l'intervention chirurgicale. Ils disposaient en permanence d'eau de boisson. La composition du régime expérimental est donnée au tableau 1. L'ingestion d'aliment a été contrôlée chaque jour de la semaine et l'évolution du poids vif des lapins a été suivie par des pesées au moins 2 fois par semaine.

TABLEAU 1

Composition de l'aliment

\begin{tabular}{|c|c|c|c|}
\hline Composants & p. 100 & Teneurs & p. 100 \\
\hline $\begin{array}{l}\text { Blé } \ldots \ldots \ldots \ldots \\
\text { T, de tournesol } \ldots \ldots \ldots \ldots \ldots \ldots \\
\text { Luzerne déshydratée } \ldots \ldots \ldots \ldots \\
\text { Complément minéral }{ }^{\prime} \text { et vitami- } \\
\text { nique } \ldots \ldots \ldots \ldots \ldots \ldots \ldots \ldots \ldots \ldots\end{array}$ & $\begin{array}{r}37 \\
9 \\
50 \\
4\end{array}$ & 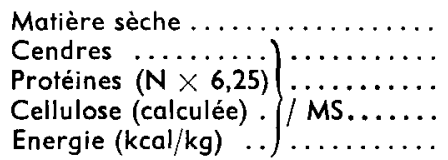 & $\begin{array}{r}89,5 \\
10,5 \\
19,7 \\
17,6 \\
4291\end{array}$ \\
\hline
\end{tabular}

Ligature du canal pancréatique. - Treize jours après leur entrée en cage, alors qu'ils étaient accoutumés à la cage et au régime alimentaire, les lapins ont été divisés en 2 lots de huit, de même poids vif moyen et de sexes également répartis ( 50 et $3 q$ par lot). Le premier lot (lot $\mathrm{P}^{-}$- poids vif moyen : $1,39 \pm 0,05 \mathrm{~kg}$ ) a subi une ligature du canal pancréatique 14 jours après l'entrée en cage, donc à l'âge de 51 jours. Le deuxième lot (lot TO — poids vif moyen : $1,41 \pm 0,06 \mathrm{~kg}$ ) a subi le même jour une laparotomie médiane. La technique chirurgicale de ligature a été celle précédemment décrite par Corring et Lebas (1977). L'efficacité de la ligature du conduit pancréatique a été vérifiée en fin d'essai par abattage des animaux, d'après la dilatation de la lumière et l'épaississement du canal associés à l'atrophie du tissu pancréatique (Catala, 1976). 
Mesure de la digestibilité de l'aliment. — La digestibilité apparente de l'aliment a été mesurée sur chaque individu par la méthode de la collecte totale réalisée en 2 fois 4 jours répartis sur 2 semaines de calendrier, comme décrit par Colin et Lebas (1976). Pour chaque individu, 2 estimations des CUD ont été réalisées. Les collectes ont commencé 5 jours après l'opération chirurgicale pour la première et 33 jours après cette même opération pour la seconde estimation. Les coefficients d'utilisation digestive apparente (CUDa) de la matière sèche (MS), de la matière organique (MO), de l'azote $(N)$ et de l'énergie ont été calculés selon la méthodologie précédemment décrite par Lebas (1975).

Analyse mathématique des résultats. - En fonction de la nature des éléments comparés, les résultats ont été analysés par une analyse de variance à un facteur contrôlé (comparaison entre lots) ou à 2 facteurs contrôlés (évolution pour une série d'animaux donnée entre périodes successives).

\section{Résultats}

Le premier résultat concerne la mort de 3 lapins dans le lot $\mathrm{P}^{--}$, respectivement aux $30 \mathrm{e}, 33^{\mathrm{e}}$ et $41^{\mathrm{e}}$ jours après ligature du canal pancréatique. Ces animaux avaient présenté auparavant une forte diarrhée. Les résultats de digestibilité portent donc sur les 2 lots complets pour la première période de collecte (de $J_{5} \grave{a}_{16}$ post-opératoire) et sur 8 animaux dans le lot témoin (effectif initial) et 5 animaux dans le lot $\mathrm{P}-$ pour la deuxième période de collecte $\left(J_{33}\right.$ à $J_{44}$ post-opératoire).

Digestibilités apparentes. - Les divers coefficients de digestibilité apparente déterminés au cours des deux périodes de collecte sont rapportés au tableau 2 . Pour la

TABLEAU 2

Digestibilité de l'aliment au cours de deux périodes post-opératoires

\begin{tabular}{|c|c|c|c|}
\hline \multirow{2}{*}{\multicolumn{2}{|c|}{ CUDa }} & \multicolumn{2}{|c|}{ Périodes } \\
\hline & & 5-16 jours & $33-44$ jours \\
\hline MS & $\begin{array}{l}\text { TO } \\
\mathrm{P}^{-}\end{array}$ & $\begin{array}{l}70,9 \pm 2,6 a \\
66,2 \pm 0,5 a\end{array}$ & $\begin{array}{l}68,5 \pm 0,7 a \\
69,6 \pm 4,0 a\end{array}$ \\
\hline MO & $\begin{array}{l}\text { TO } \\
\mathrm{P}\end{array}$ & $\begin{array}{l}69,7 \pm 3,1 a \\
66,1 \pm 1,0 a\end{array}$ & $\begin{array}{l}66,2 \pm 0,8^{a} \\
68,1 \pm 4,6 a\end{array}$ \\
\hline Energie & $\begin{array}{l}\text { TO } \\
\text { P- }\end{array}$ & $\begin{array}{l}66,2 \pm 3,4^{a} \\
63,9 \pm 0,8 a\end{array}$ & $\begin{array}{l}66,6 \pm 0,8 a \\
67,0 \pm 4,7 a\end{array}$ \\
\hline Azote & $\begin{array}{l}\text { TO } \\
\text { p- }\end{array}$ & $\begin{array}{r}86,1 \pm 2,7 a \\
\left({ }^{1}\right) * 64,9 \pm 5,2^{a}\end{array}$ & $\begin{array}{r}92,3 \pm 0,7 b \\
\text { (1) } * * 86,7 \pm 1,0^{b}\end{array}$ \\
\hline
\end{tabular}

(1) Les CUDa pour $\mathrm{P}^{-}$et TO diffèrent au seuil : * $\mathrm{P}<0,01 ; * * \mathrm{P}<0,001$.

$a, b$ : Sur une même ligne, les valeurs ayant la même lettre en indice ne sont pas significativement différentes au seuil $P<0,05$.

MS : Matière sèche ; $M O$ : Matière organique. 
$1^{\text {re }}$ période de collecte, les CUDa de la matière sèche et de l'énergie sont légèrement inférieurs chez les animaux à conduit pancréatique ligaturé par rapport à ceux des lapins témoins (respectivement $-4,7$ et $-2,6$ points), mais ces différences ne sont pas significatives. De plus elles ont disparu lors de la $2^{\mathrm{e}}$ période de mesure située 4 semaines plus tard.

Par contre, le CUDa de l'azote est très fortement réduit par la ligature du canal pancréatique dès la première période de collecte située en moyenne 10 jours après l'opération (-21,2 points ; $P<0,01)$. Par la suite, le CUDa de l'azote s'améliore très significativement dans le lot $P^{-}$et lors de la seconde période de mesure, l'écart $n^{\prime}$ 'est plus que de $-5,6$ points par rapport au lot témoin. Il faut en effet estimer les CUDa du lot $\mathrm{P}^{-}$par rapport à ceux du lot TO et non dans l'absolu puisque le CUDa de l'azote évolue significativement avec l'âge dans le lot témoin ( $+6,2$ points). Ainsi, lors de la 1 re période, le CUDa de l'azote pour le lot $P$ - n'était que de 75,4 p. 100 de celui du lot TO ; un mois plus tard, cette proportion est remontée à 93,9 p. 100.

Consommation alimentaire et croissance. - L'évolution de la consommation alimentaire et celle du gain de poids des animaux sont consignées dans les figures 1 et 2 . Dès le $2^{\mathrm{e}}$ jour post-opératoire, les lapins des deux lots ont au moins retrouvé leur poids

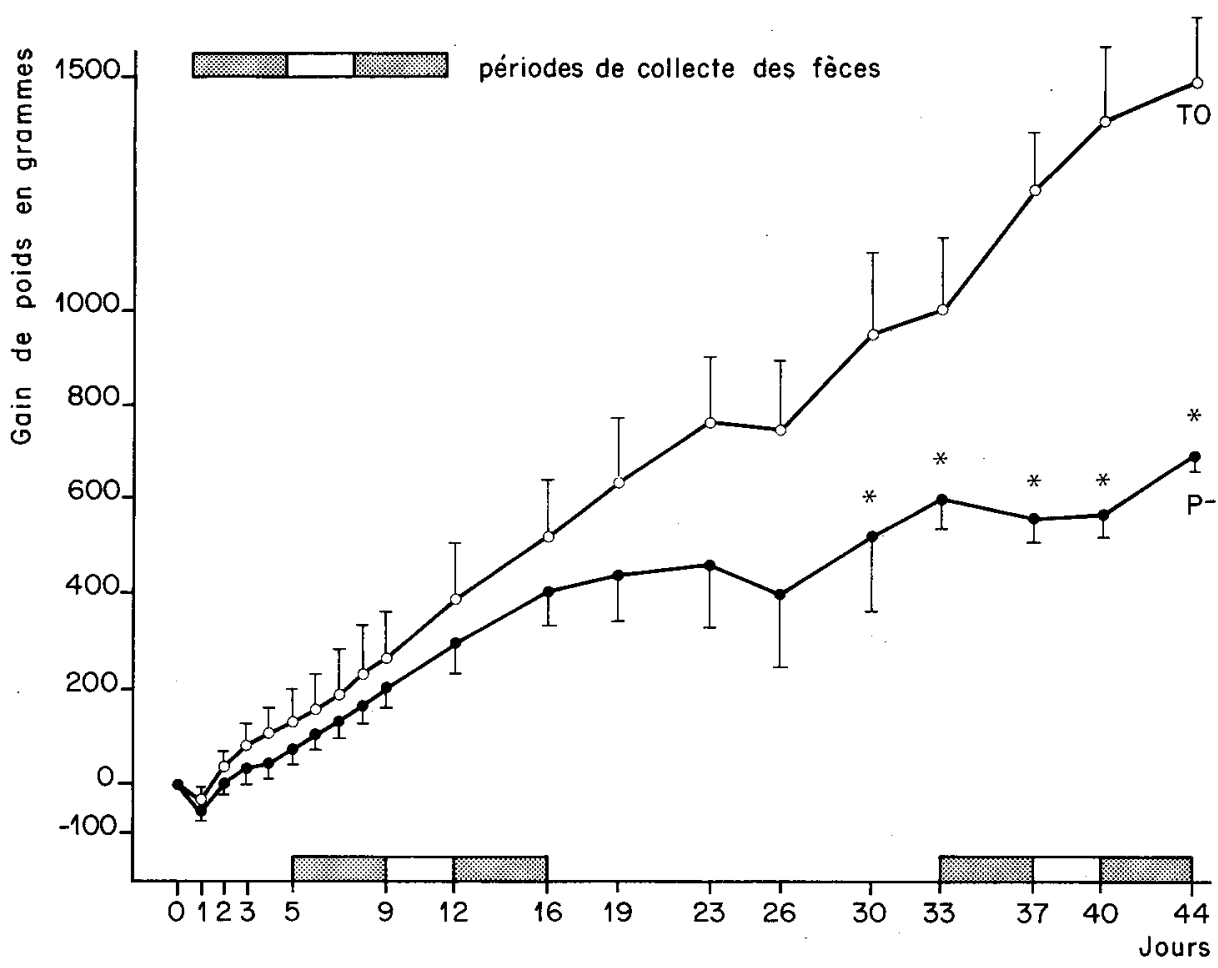

FIG. 1. - Gains de poids cumulés des lapins $p^{-}$et TO.

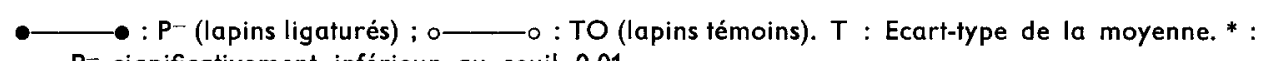

$P^{-}$significativement inférieur au seuil 0,01 . 


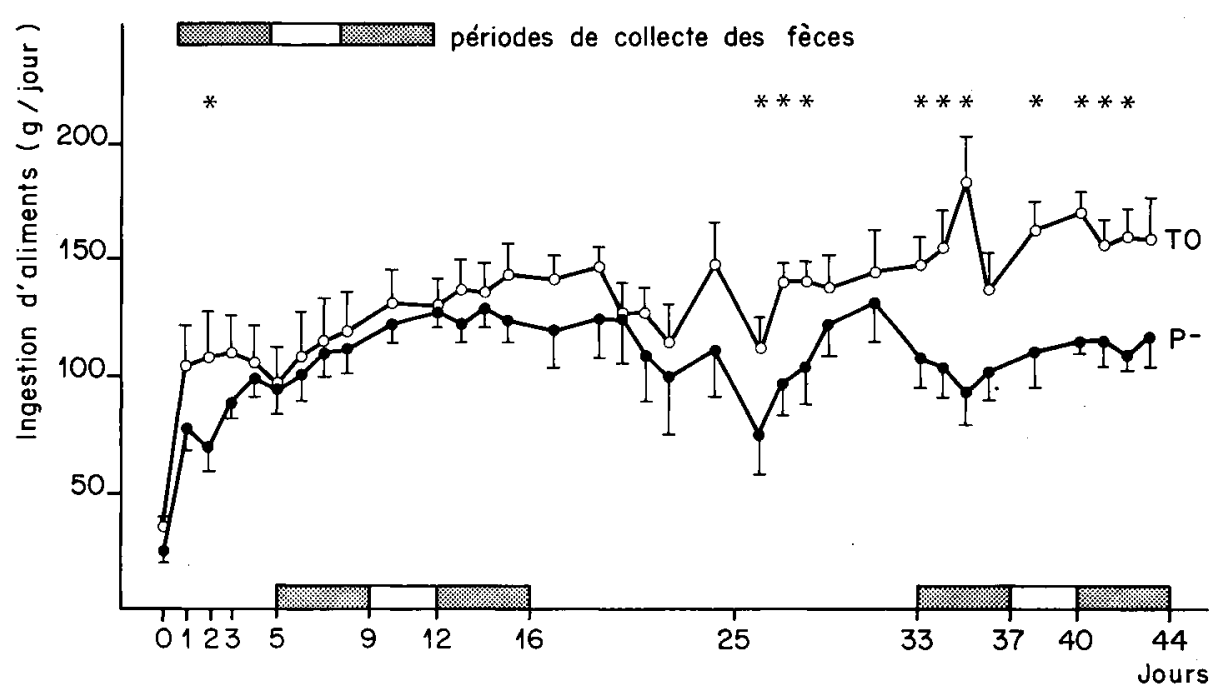

FIG. 2. - Consommation alimentaire des lapins $P-$ et TO.

$\bullet-\mathrm{P}^{-}$(lapins ligaiurés) ; o—— : TO (lapins témoins). T : Ecart-type de la moyenne. ${ }^{*}$ :

$P^{-}$significativement inférieur à TO au seuil 0,01 .

initial. Pendant les deux premières semaines, les lapins des 2 lots ont une consommation et une croissance semblables. En effet, la ligature du canal pancréatique pour le lot $\mathbf{P}^{-}$n'ayant pas entraîné de réduction significative de la digestibilité de l'énergie (tabl. 2), les animaux ont, une fois surmonté le choc opératoire, consommé les mêmes quantités d'aliment que ceux du lot TO. Compte tenu des réserves des animaux et de la forte teneur en protéines brutes de l'aliment, l'altération de la digestibilité des protéines n'a pas entraîné de réduction de la vitesse de croissance.

Par contre, au-delà du $15^{\mathrm{e}}$ jour, la consommation des animaux $\mathrm{P}^{-}$tend à se stabiliser aux environs de 100 à $120 \mathrm{~g} /$ jour alors que celle des lapins TO continue à crôitre pour atteindre $160 \mathrm{~g} / \mathrm{jour}$. Ainsi, au cours des 2 dernières semaines, l'ingestion quotidienne des lapins TO représente $202 \pm 0,6 \mathrm{kcal}$ d'énergie digestible par $\mathrm{kg}$ de poids métabolique $\left(P^{0,75}\right)$, et seulement $162 \pm 7 \mathrm{kcal}$ pour les lapins $P-(P<0,01)$. Durant la même période, la croissance des lapins $\mathrm{P}^{-}$est fortement ralentie. Compte tenu de cette évolution, la croissance globale des lapins $P$ - sur l'ensemble de la période expérimentale est moitié de celle observée pour les lapins TO (15,6 $\pm 0,6$ vs $33,7 \pm 3,5 \mathrm{~g} /$ jour, $P<0,01)$.

\section{Discussion et conclusion.}

La ligature du canal pancréatique entraîne dans les jours qui suivent une très forte réduction de la digestibilité de l'azote (-25 p. 100) chez les lapins alimentés à volonté 
comme chez les lapins rationnés (- 28 p. 100) (Corring et Lebas, 1977). Cette situation nous paraît normale puisque les consommations alimentaires quotidiennes n'ont pas différé entre lots dans aucune des deux expériences. Dans l'étude précédente (Corring et Lebas, 1977) la ligature avait affecté significativement le CUDa de l'énergie (-9,9 p. 100) chez les lapins rationnés. Dans l'étude rapportée ici, la digestibilité apparente de l'énergie subit une légère diminution (-4p. 100) non significative, ce qui laisserait à penser que les lapins nourris à volonté n'ont pas ajusté leur consommation après suppression de l'hydrolyse enzymatique.

Ce qu'il nous paraît plus important de souligner, c'est la remontée considérable du CUDa de l'azote, 5 semaines après ligature. Celui-ci ne représente alors plus qu'un écart de 6 p. 100 par rapport à celui des animaux témoins. L'origine de cette importante amélioration de la digestibilité de l'azote ne peut être précisée par les résultats obtenus dans l'étude rapportée. On peut rejeter l'hypothèse d'un refour du pancréas à un fonctionnement normal. En effet, cela pourrait être le fait d'un canal excréteur accessoire devenu fonctionnel après ligature du conduit principal. Mais ce fait paraît improbable avec un synchronisme tel que le coefficient de variation du CUDa de l'azote observé pour les 5 lapins $\mathrm{P}^{-}$, soit seulement de 2,7 p. 100. Enfin, dans les travaux antérieurs où la ligature a été réalisée comme dans notre cas, à moins de $5 \mathrm{~mm}$ de la jonction du canal pancréatique ef du duodénum, jamais une sécrétion par un canal annexe n'a été décrite (Bacques, Demigné et Vaiton, 1970 ; Catala, 1976). Seuls Avarnitakis et Folscroft (1978) mentionnent la présence de trypsine et chymotrypsine dans la lumière intestinale après ligature du canal pancréatique, mais dans leur cas, la ligature avait été opérée en un point plus éloigné du duodénum et avait parfaitement pu laisser « en circuit » une petite partie du pancréas, très diffus chez le lapin, partie qui se serait hypertrophiée par la suite.

Pour expliquer cet accroissement du CUDa de l'azote, on peut envisager soit une augmentation de l'activité protéolytique gastrique, soit plus vraisemblablement une augmentation de l'activité enzymatique protéolytique de la flore digestive cæcale observée par Catala dans des circonstances comparables (Catala et Bonnafous, 1974 ; Catala, 1978). En raison de la disparition des enzymes protéolytiques d'origine pancréatique, un flux plus important de protéines non digérées entre dans le cæcum. Les bactéries présentes dans cel organe utilisent ces protéines à leur usage propre soit comme source azotée, soit comme source énergétique après désamination. A l'appui de cefte dernière hypothèse, nous devons retenir la très faible valeur biologique de la fraction azotée apparemment digérée par les lapins $P^{-}$en fin d'expérience, l'azote ammoniacal produit passant aisément dans le sang. En effet, au cours des 2 dernières semaines, alors que les lapins témoins réalisent un gain de poids vif de $9,4 \mathrm{~g}$ par $\mathrm{g}$ d'azote digéré, les lapins à pancréas ligaturés n'ont qu'un gain de $3,9 \mathrm{~g}$ pour la même quantité d'azote passant dans la circulation sanguine. L'explication la plus vraisemblable est qu'une part importante de cet azote est sous forme ammoniacale, donc inutilisable pour le métabolisme azoté du lapin et éliminée de l'organisme dans les urines après transformation en urée. Ceci est d'ailleurs en accord avec le mauvais état sanitaire des lapins $\mathrm{P}^{-}$et la perte de 3 individus par diarrhée, phénomène classiquement provoqué par un excès d'azote ammoniacal ou uréique au niveau sanguin (Renault, 1975).

Si cette hypothèse est exacte - une vérification directe est possible en mesurant 
l'excrétion urinaire - il convient encore d'expliquer pourquoi une telle désamination n'a pas eu lieu quand les lapins étaient alimentés en quantité limitée. Il nous faut pour cela faire appel d'une part à la teneur en azote de l'aliment expérimental, d'autre part au rythme d'ingestion. En effet, bien que la formule et la nature des matières premières composant la ration expérimentale soient les mêmes dans les 2 essais (Corring et Lebas, 1977 ; et présente étude), des variations dans l'origine des lots de matières premières ont entraîné la distribution aux animaux rationnés d'un aliment contenant 9,8 p. 100 de protéines brutes digestibles par les lapins non privés de sécrétion pancréatique. Par contre, les lapins nourris à volonté ont reçu un aliment en contenant 15,8 p. 100. Proportionnellement aux autres composants, les protéines représentent une source d'énergie mise à la disposition des bactéries cæcales beaucoup plus importante dans l'expérience concernant les lapins ligaturés nourris à volonté que dans celle où les sujets sont nourris en quantité limitée. Il est donc vraisemblable que les désaminations y jouent un plus grand rôle. D'autre part, et de manière indépendante, la restriction alimentaire accroît de manière significative la vitesse d'ingestion de l'aliment (Lebas et Laplace, 1975). De ce fait, les aliments non digérés arrivent en masse dans le cæcum, et il est logique de penser que dans ces conditions, la désamination bactérienne n'est pas aussi complète que lorsque le flux alimentaire arrive plus régulièrement dans le cæcum. A l'appui de cet ensemble d'hypothèses justifiant les différences entre les lapins alimentés à volonté et ceux qui sont rationnés, il convient de signaler un mois et demi après ligature la faible différence entre la valeur biologique des protéines digestibles chez les lapins ration nés : 7,8 $\mathrm{g}$ de gain par $\mathrm{g}$ d'azote digéré pour les animaux témoins et seulement $6,1 \mathrm{~g}$ pour les animaux ligaturés, soit un écart de $22 \mathrm{p} .100$, alors que dans le cas des lapins alimentés à volonté, l'écart est de 59 p. 100 comme il a été indiqué plus haut.

Dans la justification de la mise en place du présent travail, il y avait, nous venons de le voir, la vérification de l'effet de la ligature du canal pancréatique sur la digestibilité des aliments chez le lapin alimenté à volonté. Nous nous étions également posé la question de savoir si, face à une digestion déficiente réduisant le flux de nutriments entrant dans la circulation sanguine, les lapins nourris à volonté « savaient » augmenter leur consommation pour maintenir leur croissance. L'expérience nous montre que les premiers jours suivant la ligature, les lapins alimentés à volonté maintiennent bien une consommation alimentaire comparable à celle des témoins; mais en aucun cas elle ne lui est supérieure. De plus, après quelques semaines, la quantité d'aliment ingéré quotidiennement est réduite par rapport à celle des animaux témoins et cette sousconsommation est toujours observée si on exprime les ingestions en énergie digestible par $\mathrm{kg}$ de poids métabolique. La ligature affectant essentiellement la digestion azotée (quantité et qualité de la fraction passant dans le sang), elle peut être considérée comme équivalente à une réduction de la proportion et de la qualité des protéines de l'aliment. Dans ces conditions, il est logique de constater une réduction de la consommation alimentaire et de la croissance comme cela a été observé par Lebas (1973) quand la teneur en protéines brutes de l'aliment est réduite en dessous de 17 p. 100 ou par Colin (1974) quand la composition en acides aminés est défavorable. 


\section{Références}

ARVANITAKIS C., FOLSCROFT J., 1978. Effect of pancreatic duct ligation on exocrine pancreatic function and structure in the rabbit. Experientia, 34, 77-79.

BACQUES C., DEMIGNÉ C., VAITON C., 1970. Influence de la ligature du canal pancréatique et de la cæcumectomie sur l'excrétion fécale des lipides chez le lapin. C. R. Soc. Biol. Fr., 164, 15001504.

BOMBEKE A., OKERMAN F., NORMANS R., 1978. L'influence de la granulation à sec et à la vapeur des rations à teneurs différentes en énergie sur les résultats de production des lapins de chair. Rev. Agric., 31, 945-955.

CATALA J., 1976. Contrôle de l'efficacité de la ligature du canal pancréatique chez le lapin. Ann. Rech. vétér., 7, 33-38.

CATALA J., 1978. Recherches sur la physiologie digestive chez le lapin par une éfude expérimentale de lo fonction pancréatique. Th. Doct. Etat, Univ. P. Sabatier de Toulouse, $284 \mathrm{PP}$.

CATALA J., BONNAFOUS R., 1974. Variations quantitatives de la microflore intestinale du lapin après ligature du canal pancréatique. C. R. Acad. Sci. Paris, Sér. D, 278, 3351-3353.

COLIN M., 1974. Supplémentation en lysine d'un régime à base de tourteau de sésame chez le lapin. Effets sur les performances de croissance et le bilan azoté estimé par 2 méthodes. Ann. Zootech., 23, 119-132.

COLIN M., LEBAS F., 1976. Méthodes d'étude de la digestibilité des aliments chez le lapin. II. Périodicité des récoltes. Sci. Tech. Anim. Lab., 1, 129-133.

CORRING T., BOURDON D., 1976. Short-dated influence of removal of the exocrine pancreatic secretion (enzymatic or total) upon the apparent digestibility of a diet in the pig. Nutr. Rep. int., $14,621-627$.

CORRING T., BOURDON D., 1977. Exclusion of pancreatic exocrine secretion from intestine in the pig : existence of a digestive compensation. J. Nutr., 107, 1216-1221.

CORRING T., LEBAS F., 1977. Effets d̀ court et moyen terme de la ligature du canal pancréatique sur la digestibilité d'un aliment, chez le lapin en croissance et soumis à une alimentation restreinte. Ann. Biol. anim. Bioch. Biophys., 17, 299-307.

DEHALLE C., 1980. Concentration en énergie et protéines digestibles des aliments. Effets sur les performances de croissance du lapin. Mém. 2e Congr. mond. Cuniculture (Barcelone, avril 1980), Vol. 2, 56-64.

DOUGLAS G. J., REINAEUR A. J., BROOKS W. C., PRATT J. H., 1953. The effect on digestion and absorption of excluding the pancreatic juice from the intestine. Gastroenterology, 23, 452-459.

LEBAS F., 1973. Variations chez le lapin des coefficients d'utilisation digestive de la matière sèche, de la matière organique et de l'azote en fonction de l'âge, de la race et du sexe. Ann. Biol. onim. Bioch. Biophys., 13, 767-768.

LEBAS F., 1975. Influence de la teneur en énergie de l'aliment sur les performances de croissance chez le lapin. Ann. Zootech., 24, 281-288.

LEBAS F., LAPLACE J. P., 1975. Le transit digestif chez le lapin. V. - Evolution de l'excrétion fécale en fonction de l'heure de distribution de l'aliment et du niveau de rationnement durant les 5 jours qui suivent l'application de ce dernier. Ann. Zootech., 24, 613-627.

RENAULT L., 1975. La pathologie digestive du lapin. In : Le lapin, règles d'hygiène et d'élevage. Inf. tech. Serv. vet., 51 à 54, 129-141.

SATERI H., 1975. Investigations of the exocrine pancreatic function in dogs suffering from chronic exocrine pancreatic insufficiency. Acto vef. scand., suppl. 53, 1-86.

SHINGLETON W. W., WELLS M. H., BAYLIN G. H., RUFFIN J. M., SAUNDERS A., DURHAM N. C., 1955. The use of radioactive labelled protein and fat in the evaluation of pancreatic disorders. Surgery, 38, 134-142.

SPREADBURY D., DAVIDSON J., 1978. A study of the need for fibre by the growing New Zealand white rabbit. J. Sci. Fd. Agric., 29, 640-648. 